\title{
Scientific Summary of the First BINA Workshop
}

\author{
Ram Sagar *
}

Indian Institute of Astrophysics, Bangalore, 560034, India

\begin{abstract}
Scientific summary of the first Belgo-Indian Network for Astronomy \& Astrophysics (BINA) workshop is presented here. In the workshop, invited talks were supplemented with the contributory talks and poster paper presentations. The talks presented during the workshop provide latest information on the 3.6-m Devasthal Optical Telescope (DOT) and 4-m International Liquid Mirror Telescope (ILMT) projects.These observing facilities are built jointly by the astronomical communities of India and Belgium. In the ILMT, reseachers from Canada, Poland and Uzbekistan are also actively participating. Preliminary results obtained from the on sky test indicate that 3.6-m DOT is capable of resolving binary stars separated by $\sim 0.4$ arc second. CCD observations obtained with the telescope indicate that atmospheric conditions at Devasthal are very good for optical observations and the site can be considered internationally competitive. Buildings and telescope houses constructed at Devasthal have not deteriorated natural seeing conditions observed at the site $\sim 2$ decades ago during 19971999. Pointing and tracking accuracies of the 3.6-m DOT are as per specifications. All these indicate that on sky performance of the newly installed 3.6-m DOT is better than the specifications prescribed at the time of placing order. It is therefore capable of providing internationally competitive science with the modern backend instruments and also have global importance due to their geographical location particularly for the time domain and multi-wavelength astrophysical studies. A large number of talks and poster papers presented during the workshop discussed astrophysical potential of the Indian largest size (4 meter class) new technology optical telescopes located at Devasthal. All these indicate beyond doubt that there are enormous opportunities for the growth of astronomy in India.
\end{abstract}

\section{Introduction}

In order to foster interaction between the astronomical communities of the Belgium and India, a research network called Belgo-Indian Network for Astronomy \& Astrophysics (BINA) has been established recently by the funding agencies of the two countries namely Belgian Science Policy (BELSPO) and Department of Science and Technology (DST). The first BINA workshop was held in the lake city of Devbhumi, Nainital, Uttarakhand, India during 15-18 November 2016. The participants $(>100)$ in the workshop were not only from Belgium and India but also from China, Germany, Japan, Russia, South Africa, Taiwan and Thailand. This makes it a truly international scientific meeting. Out of 79 contributions made during the workshop, the number of invited talks, contributed talks and poster papers were 13, 24 and 42 respectively. Table 1 presents their ditribution in the various sessions of the workshop. Scientific areas covered under these presentations range from Solar system objects, stars, star clusters to the Galaxies with an aim to address front line astrophysical research

*ramsagar@iiap.res.in; ram_sagar0@yahoo.co.in 
Table 1: This table shows the distribution of invited talks, contributory talks and poster papers in the first BINA workshop.

\begin{tabular}{|l|c|c|c|c|}
\hline Description of the workshop sections & Invited talks & Contributed talks & Poster papers & Total \\
\hline 3.6 m DOT and back-end instruments & 4 & 0 & 1 & 5 \\
Next generation instruments on 3.6 m DOT & 2 & 2 & 2 & 6 \\
Synergy of 3.6 m DOT with space Astronomy & 1 & 0 & 0 & 1 \\
ILMT and multi-wavelength Asrophysics & 1 & 1 & 2 & 4 \\
Scientific programs \& relevant instruments & 0 & 3 & 4 & 7 \\
Variable stars & 2 & 1 & 6 & 9 \\
Open star clusters & 0 & 2 & 5 & 7 \\
Multiple stellar systems & 0 & 4 & 3 & 7 \\
Star forming regions & 0 & 1 & 6 & 7 \\
Solar system and Exo-Planets & 0 & 1 & 3 & 4 \\
Active Galactic Nuclei & 1 & 3 & 5 & 9 \\
Supernovae, Transients \& Gravitational lensing & 0 & 3 & 4 & 7 \\
Galaxies & 0 & 3 & 1 & 4 \\
Closing Session & 2 & 0 & 0 & 2 \\
\hline Total & 13 & 24 & 42 & 79 \\
\hline
\end{tabular}

problems like life-cycles of stars, search for the first stars and galaxies in the Universe, etc. On the other hand, technical presentations provided information on first science verfication results of the 3.6$\mathrm{m}$ Devasthal Optical Telescope (DOT). They also presented the astrophysical potential of both $3.6 \mathrm{~m}$ DOT and 4-m International Liquid Mirror Telescope (ILMT) and their back-end instruments.

In addition to the above mentioned scientific and technical sessions, there was a special session on future key scientific projects to be undertaken under the BINA programme. This session was chaired by the Head of the international division, DST, Government of India. There was also a public lecture on the Music of the stars. It was delivered by Prof. Chris Engelbrecht from University of Johannesburg, South Africa on November 17, 2016. On the last day of the Workshop, a good number of participants visited the 3.6-m DOT and other observing facilities located at Devasthal, Nainital. On the way to Devasthal from Nainital, participants were able to see natural beauty of the mountain terrain and snow peaks of the Himalayas.

Before providing scientific summary of the first BINA workshop, let me briefly mention about its historical background. First talk of the workshop, after inaugural function on November 15, 2016, was by B.B. Sanwal who dwelled upon the glorious history of the Aryabhatta Research Institute of Observational Sciences (ARIES). In this talk growth of the Institution under state government was presented. A detailed description of this historical aspect is given by Ramachandran (2004), Sinha (2005) and Sagar (2006). It is worth mentioning here that total funds made available to the Institution were only $\sim 295$ Million Indian Rupees during a span of $\sim 50$ years by both Uttar Pradesh (from April 20, 1954 to November 8, 2000) and Uttarakhand (from November 9, 2000 to March 21, 2004) state governments. However, this situation improved significantly after March 22, 2004 when ARIES became an autonomous research institution under DST, Government of India. More funds (average annual funding of $\sim 300$ Million Indian Rupees during 2004 to 2012) became available to ARIES and the idea of jointly developing front line major optical and near-infrared (NIR) observational facilities at Devasthal, Nainital germinated in 2005 when the present author met with the astronomical community of Belgium under leadership of Prof. Jean Surdej and Prof. Jean-Pierre Swings in Liege, Belgium. In 2006, the funding agencies DST, Government of India and BELSPO of the Belgian 


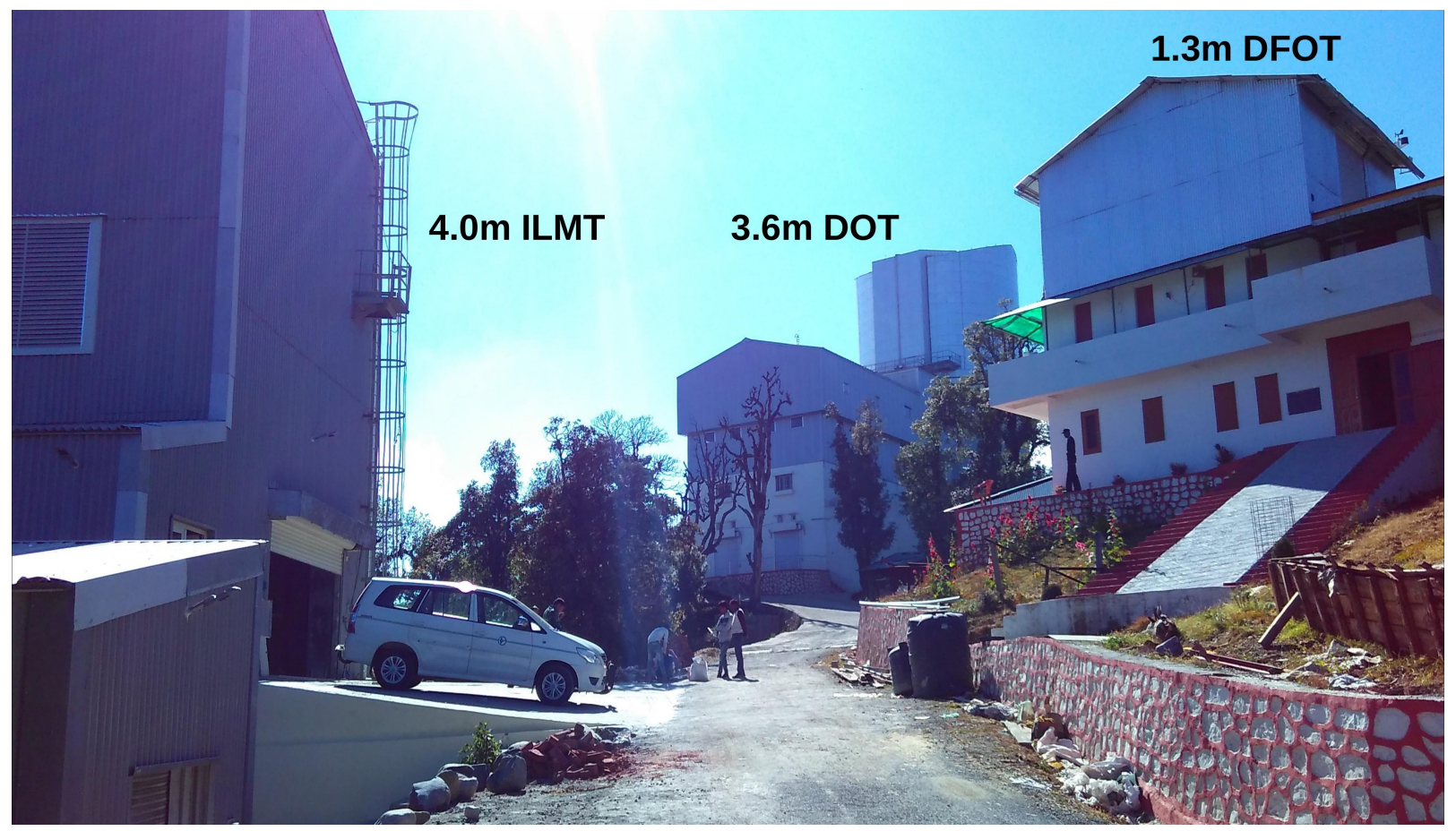

Figure 1: This figure shows the locations of the $1.3 \mathrm{~m}$ DFOT, $3.6 \mathrm{~m}$ DOT and $4 \mathrm{~m}$ ILMT telescopes near the top of the Devasthal Mountain.

Government signed an agreement for bi-lateral scientific collaboration between the scientific communities of both countries identifying Astronomy and Astrophysics as one of the key area for long term cooperation. In 2008, the BELSPO contributed 2 million Euros in cash to the 3.6-m DOT Project. In return, Belgian astronomers are assured of $7 \%$ observing time on this northern hemisphere modern $3.6 \mathrm{~m}$ DOT observing facility which was suceesfully installed in 2015 and technically activated on March 30, 2016 jointly by the Premiers of both countries, India and Belgium.

Since invited and contributed talks as well as poster paper presentations are part of this workshop proceedings, only on sky performance of the newly installed modern 3.6-m DOT as well as its astrophysical potential for the global astronomical community are highlighted in the next section of the manuscript. Status of the ILMT project is the subject matter of section 3 while synergy of 3.6-m DOT observing facility with the space astronomy is outlined in section 4. Presentations highlighting the astrophysical potential of Devasthal optical and NIR observing facilities are briefed in section 5. The last section of the manuscript presents a summary and conclusions of the presentations made during the first BINA workshop.

\section{3.6-meter DOT and its first light back-end instruments}

In the central region of Indian Himalaya, the Devasthal (longitude 79.68 deg E; latitude 29.36 deg N and altitude $2450 \mathrm{~m}$ ) was identified as a good site for optical and NIR ground based observations after an extensive characterization carried over a decade. For details, one can refer to Sagar et al. (2000) and references therein. It became an observatory in 2010 with the successful installation of the modern $130 \mathrm{~cm}$ Devasthal Fast Optical Telescope (DFOT). Its technical details and on sky performance are given by Sagar et al. (2011). Fig. 1 shows the locations of the 1.3-m DFOT, 3.6-m DOT and 4-m ILMT near the Devasthal temple, heighest location on the mountain.

Under this section, three invited talks and one poster paper were presented during the workshop. 
First science verification results from the recently installed 3.6-m DOT telescope were presented by Brijesh Kumar along with atmospheric parameters of the site characterisation work and a brief overview of installation activities. He also mentioned about the activities carried out during the installation of the alumunizing facility at the site (Reddy et al. 2016). This facility is located in the building of the telescope dome house and can aluminize mirrors upto a diameter of 3.7 meter in size. It is working satisfactorily at the site and has been used to aluminize successfully the primary mirror of the 3.6-m DOT twice first in 2015 and recently in March 2017. The modern low thermal mass telescope dome house has been built in an extremely optimized way due to availability of very limited space at the mountain top. The diameter of the dome is only 16.5 meter and the telescope center is located at a distance of 1.85 meter from the dome center. Further technical specifications of this modern and compact telescope house are described elegantly by Gopinathan et al. (2016).

There were two presentations on the first light instruments for the 3.6-m DOT namely $4 \mathrm{KX} 4 \mathrm{~K}$ CCD imager and faint object spectrograph and Camera (FOSC). Technical details and first on sky performance of the CCD imager were presented by S.B. Pandey while those of the FOSC were presented by A. Omar. CCD imager equipped with astronomical filters is now available to the astronomical community for regular optical photometric observations.

\subsection{Next generation back-end Instruments and their science driver}

It is well known fact that any optical telescope collects only photons coming from the sky. However, to understand the nature as well as the physical processes and other properties of the celestial bodies emitting the electromagnetic radiation, building of modern and state of the art back-end instruments based on latest front edge technology are essential. For this, use of expertise available in India and abroad will be extremely fruitful and valuable. Presentations made during the workshop indicate that this aspect is being seriously considered by both the Belgian and Indian astronomical communities.

Status of the proposed next generation back-end instruments on the above described 3.6-m DOT were presented in 2 invited and 2 contributed talks and in another 2 poster papers. D. K. Ojha presented the status of the 0.6 to $2.5 \mu \mathrm{m}$ Optical NIR Medium Resolution spectrograph called TANSPEC (TIFR-ARIES NIR Spectrometer) while A.N. Ramaprakash described the Devasthal Optical Telescope Integral Field Spectrograph (DOTIFS). Both instruments are being built under joint multiinstitutional collaborations. Science drivers for building these modern instruments were also presented along with their technical details and capabilities when they will be mounted on the 3.6-m DOT. Both instruments are in advanced stage of manufacturing and lab testing. They are expected to become available for regular observations to the astronomical community soon, may be within a couple of years.

M. Gopinathan discussed about a few front line and exciting science cases that can be carried out with an optical and near-infrared polarimeter mounted on the 3.6-m DOT. N. Kobayashi presented technical details of a warm high sensitivity $\operatorname{NIR}(\lambda=0.9$ to $1.35 \mu \mathrm{m})$ high resolution $(\mathrm{R}=30,000$ or $70,000)$ spectrograph as one of the best example instruments for 4-m class telescopes.

Preliminary optical designs of high resolution $(\mathrm{R}=60,000)$ spectrograph and high speed time series CCD phtometer for the 3.6-m DOT were presented in the two poster papers.

In order to exploit the full potential of the successfully installed 3.6-m DOT at geographically advantageous location (Sagar 2017; Omar et al. 2017), more complex, modern and sensitive back-end instruments based on the cutting edge technology need to be built using expertise available not only with Indian and Belgian but the entire global astronomical communities. Such instruments will make the 3.6-m DOT as one of the most productive and useful observing facilities to the global astronomers particularly for the astrophysical studies of transient objects like Supernovae and GRB afterglows, etc. This telescope will also play a major role in multi-wavelength studies of astrophysically interesting 
objects observed at radio wavelengths with the Indian Giant Meter Radio Telescope (GMRT) and at $\mathrm{X}$-ray and Ultra-violet wavelengths with the India's first multi-wavelengths unique space observatory ASTROSAT launched successfully on September 28, 2015 (Rao et al. 2016).

\section{4-meter International Liquid Mirror Telescope (ILMT)}

Installation of the 4-m ILMT at Devasthal, Nainital (see Fig. 1) will be over soon. Its present status has been elegantly described by Surdej et al. (in these workshop proceedings). This facility will be used in the time delay integration mode. Its technical details were presented by Jean Surdej while the capabilities of the prime focus $4 \mathrm{k} \mathrm{X} 4 \mathrm{~K}$ CCD imager mounted at the prime focus of the ILMT was discussed by Brajesh Kumar. Both speakers also discussed about the science driver for this unique observing facility.

Based on the time series images obtained during 2015, astrophysically important celestial objects passing through the ILMT field of view were presented in the two poster papers along with the present status of the ILMT data redution pipeline and potential of the ILMT for detecting space debris.

The above mentioned talks and poster presentations clearly point out the scientific potential of the 4-m ILMT for the detection and follow-up observations of variable celestial objects such as supernovae, variable stars and quasars, etc located in a long but narrow ( $\sim 24$ arc minute) strip of the northern hemisphere Devasthal sky passing over the ILMT field of view. For this, deep CCD photometric observations will be obtained at Zenith, the best possible location for sky observations since it has least atmospheric attenuation and best seeing conditions. The scientific output from the ILMT project is expected to be astrophysically valuable.

\section{Synergy with space astronomy}

The preliminary science results obtained from the intial performance/verification observations taken with the Ultra-violet Imaging Telescope (UVIT) onboard the first Indian Multi-wavelength satellite ASTROSAT were presented by S.K. Ghosh along with special prospective on synergy between the 3.6-m DOT and UVIT as well as other space observatories. The importance of the 3.6-m DOT for providing optical and NIR Spectroscopic and photometric observations as a part of multi-wavelength study of astrophysically interesting $\gamma$-ray, X-ray, UV and radio sources was also discussed in the talk.

It was heartening to note that all payloads on the ASTROSAT are working satisfactorily as expected from the laboratory tests on the ground. This reflects the excellent satellite launching capabilty of the Indian Space Research Organization (ISRO) built during the last few decades. A detailed report on the performance of the ASTROSAT is given by Rao et al. (2016) along with a few astrophysically interesting results. Tandon et al. (2017) have reported about the performance of the UVIT in orbit along with its potential for high angluar resolution multi-wavelengths UV observations. Using observations taken with the UVIT, Subramaniam et al. (2016) have reported the discovery of a hot companion associated with a blue straggler star in the old open star cluster NGC 188.

\section{Scientific programmes and potential of the BINA collaboration}

A large number of contributions ( 3 invited and 21 contributed talks and another 37 poster papers) described astrophysically important projects that can be undertaken using the observational facilities located at Devasthal. They covered a wide range of topics with the aim to address the origin of planetary sytems around stars; star formation and stellar evolution including probing the powerful and 
enigmatic black holes, origin of galaxies and search for the first star and galaxies in the Universe, etc. The importance of the 4-m class modern optical and NIR observational facilities located at Devasthal for the global astronomers was also highlighted for time critical observations of celestial objects like GRB afterglows and Supernovae, etc as they are located near the center of a wide longitudinal gap between Canary Island ( $\sim 20 \mathrm{deg}$ West $) /$ Caucasus mountains ( $\sim 40 \mathrm{deg}$ East $)$ and Eastern Australia $(\sim 150$ deg East). In this context, the importance of the BINA collaboration was also detailed in some of the presenations available in these proceedings of the workshop.

\section{Summary and Conclusions}

The modern thin mirror new technology 3.6-m DOT and 4-m ILMT are the largest size optical telescopes located in India. They are built under international collaboration and installed in the central Himalayan region at Devasthal in Nainital district of Devabhumi, Uttarakhand state. The place for locating these optical telescopes was chosen after an extensive site chracterization carried out using modern instruments over an extended period (Sagar et al. 2000). The recent on sky tests carried out with the 3.6-m DOT reveal that the optics of the telecope is excellent and capable of providing images of the celestial bodies with sub arc second resolutions. The best recorded value of angular resolution is 0.4 arc second (Omar et al. 2017; Sagar 2017). CCD images of a star cluster taken with the 3.6-m DOT indicate that stars fainter than 24 mag in B band are detected during an exposure time of 30 minutes which is $\sim 4$ mag fainter than the detection limit of the $104 \mathrm{~cm}$ Sampurnanand telescope of ARIES located at Manora Peak, Nainital. However, out of this 4 mag gain only 2.78 mag can be attributed to the difference in aperture sizes of the telescopes. The remaining gain in the detection limit of the 3.6-m DOT can only be attributed to the sub arc second atmospheric seeing at Devasthal even after completion of the telescope and surrounding infrastructure buildings. This as well as the CCD images taken with the 1.3-m DFOT indicate that sky conditions at Devasthal are comparable to the best locations of optical observatories on the globe. The care taken in the design and the structure of the telescope houses has paid a rich dividend as their thermal mass is so low that it has not degraded the natural atmospheric seeing at Devasthal measured $\sim 2$ decades ago during $1997-1999$ using modern differential image motion monitor (Sagar et al. 2000, and references therein).

As discussed above, the telescope collects photons while the throughput of back end instruments defines the quality of scientific output coming from the telescope. The 4-meter class modern observing facilities located at Devasthal have advantage of their geographical location for providing optical and NIR observations of not only transients objects like GRB afterglows and Supernovae but also of astrophysically significant $\gamma$-ray, X-ray, UV and radio sources observed with Indian and other observatories operating at these wavelengths. The observing facilities located at Devasthal may become of global importance when modern high resolution spectrographs equipped with most sensitive CCD detectors are mounted on the 3.6-m DOT (Sagar 2017; Omar et al. 2017). The BINA and other similar international research networks can boost the scientific output coming from these observing facilities. Such a collaboration may also provide the opportunity for developing modern back end instruments using cutting edge technology of today.

In the light of above, it can be stated with confidence that the observing facilities located at Devasthal have an enormous potential as they can provide important optical and NIR observations for a large number of front line astrophysical research problems of international importance. They can also be used for hand on manpower training of next generation. All these indicate that in the coming years, the Devasthal observatory will become well known globally. 


\section{Acknowledgements}

The author thanks the National Academy of Sciences, India (NASI) for an award of NASI-Senior Scientist Platinum Jubilee Fellowship and the Director of Indian Institute of Astrophysics, Bengaluru for providing the necessary support. Thanks to the Governing Council of ARIES and the Project Management Board of 3.6-m DOT and 4-m ILMT for providing valuable support and guidance without which these projects may have not reached to their present stage. The efforts put in by the entire staff of ARIES during the installation of these facilities at Devasthal are commendable and acknowledged with great appreciation. Invitation extended by the Director, ARIES for participation in the workshop is thankfully acknowledged. Thanks to the Scientific Organizing Committee of the workshop for providing me this academically useful opportunity.

\section{References}

Gopinathan M., Sahu S., Yadav S. et al. 2016, Proceedings of the SPIE conference, Vol. 9913, E40G

Omar A., Kumar B., Gopinathan M., Sagar R. 2017, CSci, 113, 682

Ramachandran R. 2004, Frontline published by the Hindu, 21, Issue 9 page 90

Rao A.R., Singh K.P., Bhattacharya D. 2016, SpReT, 196, 30

Reddy B.K., Gopinathan M., Pant J. et al. 2016, Proceedings of the SPIE conference, Vol. 9906, E44B

Sagar R. 2006, BASI, 34, 37

Sagar R. 2017, PINSA, 87, 1

Sagar R., Omar A., Kumar B. et al. 2011, CSci, 101, 1020

Sagar R., Stalin C. S., Pandey A. K. et al. 2000, A\&AS, 144, 349

Sinha K. 2005, Fifty Golden years (1954-2004) published by Vikrant Computers, Haldwani

Subramaniam A., Sindhu N., Tandon S. N. et al. 2016, ApJ,833, L27

Tandon S. N., Hutchings J. B., Ghosh S. K. et al. 2017, JApA, 38, Art. id 28. 